\title{
Mortality risk of long-term amiodarone therapy for atrial fibrillation patients without structural heart disease
}

\author{
Dingxin Qin, George Leef, Mian Bilal Alam, Rohit Rattan, Mohamad Bilal Munir, \\ Divyang Patel, Furqan Khattak, Evan Adelstein, Sandeep K. Jain, Samir Saba \\ Heart and Vascular Institute, University of Pittsburgh Medical Center, \\ Pittsburgh, Pennsylvania, United States
}

\begin{abstract}
Background: Amiodarone is often prescribed in the management of atrial fibrillation (AF) but is known to cause significant end-organ toxicities. In this study, we examined the impact of amiodarone on all-cause mortality in $A F$ patients with structurally normal hearts.
\end{abstract}

Methods: We performed a retrospective cohort analysis of all AF patients with structurally normal hearts who were prescribed antiarrhythmic drugs $(A A D)$ for rhythm control of $A F$ at our institution from 2006 to $2013(n=2,077)$. Baseline differences between the amiodarone (AMIO: $n=403)$ and other $A A D$ s (NON-AMIO: $n=1,674)$ groups were corrected for using propensity score matching.

Results: Amiodarone use as first-line therapy decreased significantly with a higher degree of prescriber specialization in arrhythmia management (31\%, 22\%, and 9\% for primary care physicians, general cardiologists and cardiac electrophysiologists, respectively, $p<0.001$ ). After propensity score matching, baseline comorbidities were balanced between the AMIO and NON-AMIO groups. Over a median follow-up of 28.2 months (range 6.0-100.9 months), amiodarone was associated with increased all-cause (HR 2.41, $p=0.012$ ) and non-cardiac (HR $3.55, p=0.008$ ) mortality, but not cardiac mortality. AF recurrence and cardiac hospitalizations were similar between the two study groups.

Conclusions: Amiodarone treatment of $A F$ is associated with increased mortality in patients without structural heart disease and therefore should be avoided or only used as a second-line therapy, when other AF therapies fail. Adherence to guideline recommendations in the management of AF patients impacts clinical outcome. (Cardiol J 2015; 22, 6: 622-629)

Key words: atrial fibrillation, amiodarone, outcome, mortality

\section{Editorial p. 603}

\section{Introduction}

Amiodarone is the most commonly used antiarrhythmic drug (AAD) for atrial fibrillation (AF) in the United States, accounting for $45 \%$ of AAD prescriptions each year [1]. Accumulating evidence has suggested that compared to other AADs, amiodarone is associated with increased mortality and rates of drug discontinuation due to its significant end-organ toxicities [2-5]. This is particularly important in AF patients without structural heart abnormalities in whom the use of other AADs or consideration of catheter ablation are preferred

Address for correspondence: Samir Saba, MD, FACC, FHRS, Chief, Electrophysiology Section, 200 Lothrop Street, UPMC Presbyterian, Suite B 535, Pittsburgh, PA, 15213, United States, tel: 412802 3372, fax: 412647 7979, e-mail: sabas@upmc.edu 
according to the 2006 and the recently published 2014 American Heart Association/American College of Cardiology/Heart Rhythm Society (AHA/ACC/ /HRS) AF guidelines, which classify amiodarone as a second-line therapy for $\mathrm{AF}$ in patients without structural heart disease after other AADs [6, 7]. Prescribing amiodarone as a first-line agent in this patient population is however not uncommon in "real world" clinical practice where its impact on long-term patient outcomes is unclear. We therefore sought to investigate how amiodarone impacts the long-term clinical outcomes of AF patients without structural heart disease, and whether the prescription patterns of amiodarone differ by the medical specialty of prescribing physicians.

\section{Methods}

\section{Study design}

The study is a cohort analysis comparing longterm outcomes of 2,077 consecutive paroxysmal or persistent AF patients without structural heart disease, who were prescribed amiodarone vs. other AADs at the hospitals and clinics of the University of Pittsburgh Medical Center (UPMC) from January 2006 to November 2013 with the goal of achieving rhythm control. The study was approved by the University of Pittsburgh Institutional Review Board.

Atrial fibrillation patients without structural heart disease who were prescribed Vaughan Williams class IC or class III AAD (flecainide, propafenone, sotalol, dronedarone, dofetilide or amiodarone) with treatment duration of at least 6 months were included in the study [8]. The initial query of the UPMC electronic medical record and pharmacologic database yielded 5,976 AF patients who were prescribed with Vaughan Williams class IC or class III AAD during the study period, among which 3,043 patients were excluded due to presence of structural heart disease including coronary artery disease $(n=2,139)$, congestive heart failure $(n=1,405)$, severe left ventricular hypertrophy $(n=82)$ or moderate to severe valvular heart disease $(n=642)$. Eleven patients were excluded for estimated glomerular filtration rate less than $30 \mathrm{~mL} / \mathrm{min}$ at baseline, 81 patients were excluded for previous use of AADs, and 764 patients were excluded for treatment duration of less than 6 months, yielding a final cohort of 2,077 patients. There were no missing data on any of the 2,077 patients included in the analysis.

The patients were followed from the date of first AAD prescription through the date of discontinuation of first $\mathrm{AAD}$ with prospective review of outpatient and inpatient medical records. Patients who died or were lost to follow-up during this period were censored at their date of death or last encounter. Death was ascertained through review of the medical records and the social security death index. We assigned patients in our cohort to one of two groups: amiodarone (AMIO) group, in which amiodarone was prescribed as $1^{\text {st }} \mathrm{AAD}$ for rhythm control; and non-amiodarone (NON-AMIO) group, in which AADs other than amiodarone (dronedarone, dofetilide, flecainide, propafenone or sotalol) were prescribed as $1^{\text {st }} \mathrm{AAD}$.

Demographic data was obtained from the clinical records. Information on comorbidities was generated from International Classification of Diseases, Ninth Revision, Clinical Modification (ICD-9-CM) codes in the clinical database with coding algorithms described by Quan et al. [9, 10]. The $\mathrm{CHA}_{2} \mathrm{DS}_{2}$-VASc score and Charlson comorbidity index were calculated for each patient for risk stratification [11, 12]. Information on AAD and other cardiac medications was obtained from the institutional pharmacologic database and clinical notes and orders in the electronic medical record. The primary medical specialty of AAD prescribers could be ascertained in 1,703 (82.0\%) patients (primary care physician or family doctor $[\mathrm{PCP}]=127$ [7.5\%], general cardiologist [GC] $=1,047$ [61.5\%], cardiac electrophysiologist $[\mathrm{EP}]=529[31.0 \%]$ ).

\section{Study endpoints}

The primary outcome measures were allcause mortality. The secondary outcome measures included cardiac mortality, non-cardiac mortality, $\mathrm{AF}$ recurrence, stroke, admission for any cardiac condition, and admission for AF. Causes of death were classified into cardiac and non-cardiac, and were adjudicated by review of clinical notes, death summary, and death certificates. Dates of AF recurrence were ascertained from clinical notes documenting recurrence of AF by electrocardiogram, electrocardiographic monitors, or recurrence of AF symptoms. Causes of hospital admission were adjudicated by review of admission notes.

\section{Statistical analysis}

Baseline characteristics are presented as means \pm standard deviation for continuous variables and as occurrence rates for dichotomous variables and were compared using the student's $\mathrm{t}$ and $\chi^{2}$ tests, respectively. To adjust for differences in baseline characteristics, propensity score matching was performed. Unbalanced baseline characteristics (age, sex, $\mathrm{CHA}_{2} \mathrm{DS}_{2}$-VASc score, 
Table 1. Baseline characteristics.

\begin{tabular}{|c|c|c|c|}
\hline & Amiodarone & Non-amiodarone & $\mathbf{P}$ \\
\hline Total & 403 & 1,674 & \\
\hline Mean age & $74.1 \pm 12.0$ & $66.4 \pm 12.5$ & $<0.001$ \\
\hline Sex (male) & $183(45.4 \%)$ & $925(55.3 \%)$ & $<0.001$ \\
\hline $\mathrm{CHA}_{2} \mathrm{DS}_{2}$-VASC score: & & & $<0.001$ \\
\hline $0-2$ & $138(34.2 \%)$ & $1,008(60.2 \%)$ & \\
\hline $3-5$ & $231(57.3 \%)$ & $597(35.7 \%)$ & \\
\hline $6-9$ & $34(8.4 \%)$ & $69(4.1 \%)$ & \\
\hline Mean $\mathrm{CHA}_{2} \mathrm{DS}_{2}$-VASc score & $3.14 \pm 1.59$ & $2.24 \pm 1.63$ & $<0.001$ \\
\hline Mean Charlson comorbidity index & $1.49 \pm 1.73$ & $0.95 \pm 1.39$ & $<0.001$ \\
\hline Hypertension & $281(69.7 \%)$ & $970(58.0 \%)$ & $<0.001$ \\
\hline Valvular heart disease (mild) & $97(24.1 \%)$ & $203(12.1 \%)$ & $<0.001$ \\
\hline Ventricular tachycardia & $4(1.0 \%)$ & $23(1.4 \%)$ & 0.544 \\
\hline Atrial flutter & $25(6.2 \%)$ & $116(6.95)$ & 0.603 \\
\hline Diabetes mellitus & $75(18.65)$ & $251(15.05)$ & 0.073 \\
\hline Hyperlipidemia & $225(55.8 \%)$ & $808(48.3 \%)$ & 0.006 \\
\hline Chronic obstructive pulmonary disease & $39(9.7 \%)$ & $99(5.9 \%)$ & 0.006 \\
\hline Chronic kidney disease & $47(9.3 \%)$ & $54(3.4 \%)$ & $<0.001$ \\
\hline Cancer & $55(13.7 \%)$ & $159(9.5 \%)$ & 0.014 \\
\hline \multicolumn{4}{|l|}{ Medications at baseline: } \\
\hline Anticoagulation & $274(68.0 \%)$ & $1,167(69.7 \%)$ & 0.500 \\
\hline Aspirin & $248(61.5 \%)$ & $1,061(63.4 \%)$ & 0.491 \\
\hline ACEI/ARB & $208(51.6 \%)$ & $785(46.9 \%)$ & 0.089 \\
\hline Beta-blocker & $265(65.3 \%)$ & $878(52.5 \%)$ & $<0.001$ \\
\hline Calcium channel blocker & $170(42.2 \%)$ & $643(38.4 \%)$ & 0.164 \\
\hline Statins & $192(47.6 \%)$ & $769(45.9 \%)$ & 0.538 \\
\hline
\end{tabular}

ACEI — angiotensin converting enzyme inhibitor; ARB - angiotensin receptor blockers

Charlson comorbidity index, hypertension, diabetes, hyperlipidemia, mild valvular heart disease, ventricular tachycardia, cerebrovascular disease, chronic obstructive pulmonary disease [COPD], chronic kidney disease, liver disease, and cancer) were included as covariates in the propensity score model. Patients were then matched on a 1:1 basis between the two study groups using a caliper width of 0.10 of the logit of the propensity score. After propensity score matching, baseline characteristics between patients in each group were calculated and compared using the Student's $t$ and $\chi^{2}$ tests. Kaplan-Meier curve was constructed for overall survival and were compared using the Cox-proportional hazard test. To assess the validity of our results, analyses of all end points were repeated using all patients by fitting Cox proportional hazard models with all baseline characteristics as covariates and then again with propensity scores entered as covariates. All tests were 2 -tailed, and p-values
$<0.05$ were considered statistically significant. All statistical analyses were performed using STATA 12.1 (StataCorp LP, College Station, Texas).

\section{Results}

\section{Study population}

The study cohort consisted of 2,077 AF patients, of whom 403 patients were in the AMIO group and 1,674 patients were in the NON-AMIO group. The median follow-up time was 28.2 (range 6.0-100.9) months, 22.4 (range 6.1-100.0) months in the AMIO group and 30.1 (range 6.0-100.9) months in the NON-AMIO group $(\mathrm{p}<0.001)$. In the AMIO group, the mean maintenance dose of the medication was $202 \pm 49$ (range 100-400) $\mathrm{mg}$ /day. The overwhelming majority (89\%) of patients were taking $200 \mathrm{mg}$ /day. Table 1 compares the baseline characteristics of the study groups. Patients in AMIO group were generally older, more likely to 
Table 2. Baseline characteristics after propensity score matching.

\begin{tabular}{|c|c|c|c|}
\hline & Amiodarone & Non-amiodarone & $\mathbf{P}$ \\
\hline Total & 403 & 403 & \\
\hline Mean age & $74.1 \pm 12.0$ & $74.3 \pm 10.9$ & 0.771 \\
\hline Sex (male) & $183(45.4 \%)$ & $163(40.5 \%)$ & 0.155 \\
\hline $\mathrm{CHA}_{2} \mathrm{DS}_{2}$-VASC score: & & & 0.617 \\
\hline $0-2$ & $138(34.2 \%)$ & $129(32.0)$ & \\
\hline $3-5$ & $231(57.3 \%)$ & $233(57.8)$ & \\
\hline $6-9$ & $34(8.4 \%)$ & $41(10.2)$ & \\
\hline Mean $\mathrm{CHA}_{2} \mathrm{DS}_{2}$-VASc score & $3.14 \pm 1.59$ & $3.19 \pm 1.68$ & 0.698 \\
\hline Mean Charlson comorbidity score & $1.49 \pm 1.73$ & $1.47 \pm 1.78$ & 0.857 \\
\hline Hypertension & $281(69.7 \%)$ & $276(68.5 \%)$ & 0.703 \\
\hline Valvular heart disease (mild) & $97(24.1 \%)$ & $93(23.1 \%)$ & 0.740 \\
\hline Ventricular tachycardia & $4(1.0 \%)$ & $5(1.2 \%)$ & 0.737 \\
\hline Atrial flutter & $25(6.2 \%)$ & $14(3.55)$ & 0.071 \\
\hline Diabetes mellitus & $75(18.65)$ & $69(17.15)$ & 0.581 \\
\hline Hyperlipidemia & $225(55.8 \%)$ & $232(57.65)$ & 0.619 \\
\hline Chronic obstructive pulmonary disease & $39(9.75 \%)$ & $46(11.45 \%)$ & 0.422 \\
\hline Cancer & $55(13.7 \%)$ & $57(14.1 \%)$ & 0.839 \\
\hline \multicolumn{4}{|l|}{ Medications at baseline: } \\
\hline Anticoagulation & $274(68.0 \%)$ & $287(71.2 \%)$ & 0.319 \\
\hline Aspirin & $248(61.5 \%)$ & $247(61.3 \%)$ & 0.942 \\
\hline ACEI/ARB & $208(51.6 \%)$ & $194(48.1 \%)$ & 0.324 \\
\hline Beta-blocker & $265(65.3 \%)$ & $183(45.4 \%)$ & $<0.001$ \\
\hline Calcium channel blocker & $170(42.2 \%)$ & $148(36.7 \%)$ & 0.113 \\
\hline Statins & $192(47.6 \%)$ & $208(51.6 \%)$ & 0.260 \\
\hline
\end{tabular}

ACEI - angiotensin converting enzyme inhibitor; ARB — angiotensin receptor blockers

Table 3. Prescribers of $1^{\text {st }}$ antiarrhythmic drugs.

\begin{tabular}{lccc}
\hline & Amiodarone & Non-amiodarone & Total \\
\hline Primary care physician & $39(30.7 \%)$ & $88(69.3 \%)$ & 127 \\
General cardiologist & $227(21.7 \%)$ & $820(78.3 \%)$ & 1,047 \\
Electrophysiologist & $47(8.9 \%)$ & $482(91.1 \%)$ & 529 \\
Total & 313 & 1,390 & 1,703 \\
\hline
\end{tabular}

be female, with higher $\mathrm{CHAD}_{2} \mathrm{DS}_{2}$-VASc score, higher Charlson comorbidity index, and higher rates of hypertension, mild valvular heart disease, hyperlipidemia, chronic obstructive lung disease, chronic kidney disease and cancer. Rates of anticoagulation were similar between the two groups. Propensity score matching produced 403 patient pairs. After propensity score matching, age and all baseline comorbidities were balanced between the two groups (Table 2).

\section{Prescribers of antiarrhythmic drugs}

Prescribers of AADs were identified in 1,703 patients, including $313(77.7 \%)$ patients in AMIO group and 1,390 (83.0\%) patients in NON-AMIO group (Table 3 ). Compared with EP, PCP and $\mathrm{GC}$ were more likely to prescribe amiodarone as a first-line $\mathrm{AAD}$ (30.7\% PCP vs. $21.7 \% \mathrm{GC}$ vs. $8.9 \%$ $\mathrm{EP}, \mathrm{p}<0.001$ ) for AF patients without structural heart disease. 
Table 4. Major clinical outcomes for amiodarone group vs. non-amiodarone group.

\begin{tabular}{|c|c|c|c|c|}
\hline & \multicolumn{2}{|c|}{ Propensity-matched cohort } & \multicolumn{2}{|c|}{ Complete cohort } \\
\hline & Hazard ratio & $\mathbf{P}$ & Hazard ratio & $\mathbf{P}$ \\
\hline All-cause mortality & 2.41 & 0.012 & 2.13 & 0.009 \\
\hline Cardiac mortality & 1.40 & 0.527 & 1.75 & 0.293 \\
\hline Non-cardiac mortality & 3.55 & 0.008 & 2.25 & 0.021 \\
\hline Atrial fibrillation recurrence & 0.93 & 0.574 & 0.88 & 0.227 \\
\hline Stroke & 0.73 & 0.451 & 0.82 & 0.622 \\
\hline Cardiac admission & 1.13 & 0.469 & 1.02 & 0.870 \\
\hline Atrial fibrillation admission & 1.00 & 0.996 & 0.89 & 0.478 \\
\hline
\end{tabular}

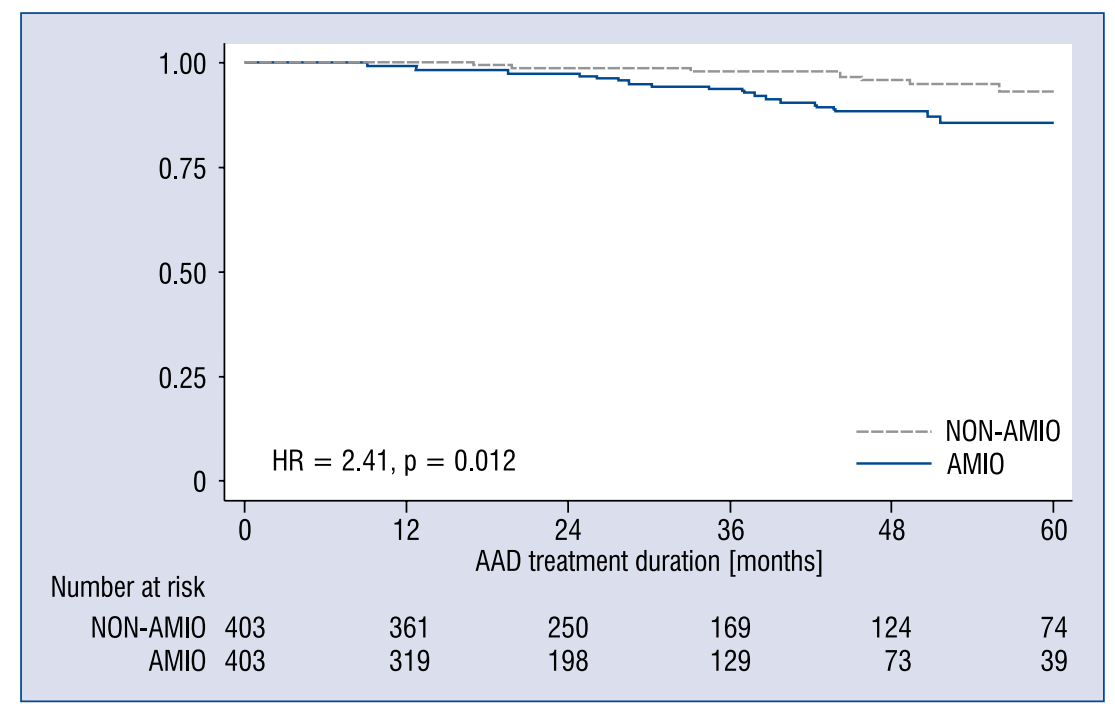

Figure 1. Overall survival for amiodarone (AMIO) group vs. non-amiodarone (NON-AMIO) group in propensity-matched cohort; AAD — antiarrhythmic drugs; HR — hazard ratio.

\section{Mortality}

In propensity-matched cohort, the AMIO group had a higher all-cause mortality rate compared to the NON-AMIO group (hazard ratio [HR] 2.41, $\mathrm{p}=0.012$, absolute crude mortality risk increased by $1.68 \%$ per year; Table 4, Fig. 1). Investigation of cause of death revealed that of the 23 deaths in the AMIO group, 7 deaths were due to cardiac causes and 16 to non-cardiac conditions. On the other hand, of the 13 deaths in the NON-AMIO group, 7 were cardiac deaths and 6 were non-cardiac deaths. The non-cardiac deaths in patients on amiodarone included respiratory failure $(n=3)$, cancer $(n=4)$, sepsis $(\mathrm{n}=3)$, pulmonary embolism $(\mathrm{n}=1)$, dementia $(n=1)$, trauma $(n=1)$, stroke $(n=1)$, and perforated bowels $(n=1)$. The cause could not be determined in 1 patient. Compared to the NON-AMIO group, the risk of non-cardiac death was significantly higher in the AMIO group (HR $3.55, \mathrm{p}=0.008$, absolute crude non-cardiac mortality risk increased by $1.49 \%$ per year; Table 4$)$. The risk of cardiac deaths was similar between the two groups (HR 1.40, $\mathrm{p}=0.527$; Table 4).

\section{Atrial fibrillation recurrence and hospitalizations}

In the propensity-matched cohort, AF recurrence occurred in 111 (27.5\%) patients in the AMIO group and $140(34.7 \%)$ patients in the NON-AMIO group during the follow-up periods. In the AMIO group, $69(17.1 \%)$ patients required admission for cardiac conditions, including $43(10.7 \%)$ patients 
with $\mathrm{AF}$ admissions. In the NON-AMIO group, 75 (18.6\%) patients had cardiac admissions, including $53(13.2 \%)$ with AF admissions. There were no significant differences in the risk of $\mathrm{AF}$ recurrences, stroke, cardiac admissions or AF admissions between the two groups (Table 4).

\section{Validation of clinical outcomes with multivariate model}

Multivariate Cox-proportional hazard analysis was performed for the complete cohort $(\mathrm{n}=$ $=2,077$ ) to validate the results from the propensity-matched cohort. In the complete cohort, there were 23 deaths in the AMIO group $(n=403)$, with 7 cardiac deaths and 16 non-cardiac deaths. Thirty-five deaths occurred in the NON-AMIO group $(\mathrm{n}=1,674)$, including 10 cardiac deaths and 25 non-cardiac deaths. After adjusting for baseline characteristics, all-cause mortality was significantly higher in the AMIO group (HR 2.13, $\mathrm{p}=0.009$; Table 4 ), which was primarily driven by the higher risk of non-cardiac death in the AMIO group (HR 2.25, $\mathrm{p}=0.021$; Table 4). No significant difference was found in cardiac mortality, stroke, $\mathrm{AF}$ recurrence, cardiac admission, or $\mathrm{AF}$ admission between the two groups.

\section{Discussion}

This cohort study suggests that compared with other Vaughan Williams class IC and class III AADs, amiodarone therapy in AF patients with structurally normal hearts is associated with a significant increase in all-cause mortality, driven primarily by higher rates of non-cardiac mortality. It also suggests that prescribing amiodarone as a first-line AAD in this patient population is more common among physicians with less specialization in arrhythmia management. These results have immediate implication for clinical practice.

Amiodarone, a complex iodinated compound, has been known for its clinical efficacy as well as its potential for serious end-organ toxicities due to tissue accumulation with long-term oral therapy, mainly in the lungs, liver, thyroid gland, nerves, skin, eyes, and heart $[2-5,13,14]$. When the Food and Drug Administration granted approval for amiodarone in 1985, it was intended for lifethreatening ventricular arrhythmias and could only be prescribed by experienced physicians [14]. Over time, however, off-label use of amiodarone became more common due to its clinical efficacy and safer pro-arrhythmic profile compared with other AADs, especially among patients with chronic heart failure or previous myocardial infarction [3, 5, 15, 16]. Analyses of the AFFIRM study suggested that among AF patients with pre-existing pulmonary disease, the use of amiodarone does not increase pulmonary death or all-cause mortality [17] and that amiodarone is superior to other AAD in maintaining sinus rhythm [18]. Presently, amiodarone is the most commonly prescribed AAD for AF [5], and has gained popularity among physicians of different medical specialties.

Conversely, there has been increasing evidence suggesting that long-term amiodarone use may increase mortality and adverse events in AF patients. In the SCD-HeFT trial, amiodarone was associated with increased non-cardiac mortality in patients with New York Heart Association heart failure class III [2]. A mixed treatment comparison analysis based on data from existing randomized control trials showed that, compared to placebo, amiodarone was associated with increased all-cause mortality (HR 2.73, p = 0.049) and increased odds of study withdrawal and drug discontinuation due to adverse side effects [3]. In a systematic review with direct comparison to dronedarone, amiodarone was associated with 9.6 more deaths and 62 more adverse events per 1,000 treated patients, resulting in discontinuation of the drug, despite lower rates of AF recurrence [4].

Our study focused on AF patients without structural heart disease, in whom the use of amiodarone can usually be avoided since other treatment options with less severe long-term side effects and toxicities are available and recommended according to published guidelines. In our cohort, amiodarone was prescribed as a first-line AAD in nearly $20 \%$ of patients, contrary to recommendations of the 2006 and $2014 \mathrm{AHA} / \mathrm{ACC} / \mathrm{HRS} \mathrm{AF}$ guideline $[6,7]$. In a propensity-matched cohort, all-cause mortality increased by 2.41 folds in the AMIO group, primarily due to a 3.55 -fold increase in non-cardiac mortality. The cardiac mortality was similar between the two groups. The etiology of the increase of non-cardiac mortality in the AMIO group may be multifold. It is well known that amiodarone toxicity, especially pulmonary toxicity, could be fatal. However, none of the patients in our cohort had pulmonary toxicity documented as the cause of death, which is consistent with the previous literature [16-19]. It is therefore likely that amiodarone contributes to worsening overall medical condition for $\mathrm{AF}$ patients, leading to higher rates of acute decompensation. For instance, although baseline COPD prevalence was similar between the two groups in the propensity-matched cohort 
(9.7\% AMIO vs. $11.5 \%$ NON-AMIO, $\mathrm{p}=0.42$ ), 5 patients in the AMIO group vs. no patients in the NON-AMIO group died of respiratory failure.

In our cohort, amiodarone was more often chosen as first-line AAD for AF by providers with less specialization in arrhythmia management. Considering the fast pace of advancement in electrophysiology, it is conceivable that primary care physicians and general cardiologists may be less up to date with the latest guidelines in AF management. Cardiac electrophysiologists, with more experience in rhythm control strategies, may also be more comfortable with prescribing other AADs with less toxicity. These considerations, which may help explain the discrepancies in use of amiodarone for $\mathrm{AF}$ by physician specialty deserve further investigation.

The results of our study suggest that amiodarone should be used with more caution in $\mathrm{AF}$ patients without structural heart disease and only after other therapies, including other AAD or ablation therapies have been tried and failed. It also suggests that strategies of rate control which have not been implicated in increased mortality rates have also to be considered when possible, particularly in asymptomatic or minimally symptomatic patients. Our results also highlight the importance of disseminating to physicians of different medical specialties knowledge regarding potential amiodarone toxicity and the availability of other rhythm control strategies. Whether more effective mechanisms for monitoring the potential side effects of amiodarone may alter patient outcomes is unclear at this point, but deserves consideration.

Our retrospective cohort study carries the inherent limitations of selection bias and information bias. To minimize the impact of selection bias, we excluded patients with structural heart disease and/or severe chronic kidney disease, so that each patient in either group would be eligible to take any of the AADs we studied. We then analyzed data with propensity-score matching methods and multivariate analysis to adjust for differences in baseline characteristics. Of note, propensity score matching can eliminate biases from observed imbalances between groups but cannot adjust for unobserved differences. In our cohort, difference in use of beta-blockers persisted between the groups despites propensity score matching. For information bias, the quality of event reporting and data collection is overall similar between the two study groups, as these data were collected simultaneously, using the same institutional electronic medical record system. For the evaluation of clinical outcomes, we focused on the mortality risk of long-term amiodarone therapy, which may not reflect the full impact of the nonfatal adverse effects associated with amiodarone. The external validity of the study may be limited by the fact that our patient population was from a single center. It is worth noting, however, that UPMC comprises a network of more than 25 hospitals as well as many outpatient clinics encompassing a large geographical area in Western Pennsylvania. With a cohort of 2,077 AF patients derived from these sites, this study has a wide representation of varying practice settings and patient demographics. Lastly, although we show in our analysis a $12 \%$ reduction in the rate of $\mathrm{AF}$ recurrence associated with the use of amiodarone, this trend did not reach statistical significance either because of lack of statistical power or because of unaccounted biases between the study groups.

\section{Conclusions}

Amiodarone use for AF is associated with increased mortality in patients with normal hearts, primarily due to increased non-cardiac deaths. It should therefore be avoided and only considered after other therapies fail. The use of amiodarone as a first-line $\mathrm{AAD}$ is more common among physicians who are less specialized in arrhythmia management, suggesting the need for better dissemination of the knowledge regarding published guideline recommendations on AF management.

Conflict of interest: Samir Saba received research support from Medtronic, Boston Scientific, and St. Jude Medical; Sandeep Jain received research support from Medtronic and Boston Scientific; Evan Adelstein received research support from Medtronic and St. Jude Medical; none of the other authors have any conflict of interest to declare.

\section{References}

1. IMS Health. National prescription audit: 2006-2010. (Extracted January 2012).

2. Marchlinski FE, Gansler TS, Waxman HL et al. Amiodarone Pulmonary Toxicity. Ann Intern Med, 1982; 97: 839-845.

3. Freemantle N, Lafuente-Lafuente $\mathrm{C}$, Mitchell $\mathrm{S}$ et al. Mixed treatment comparison of dronedarone, amiodarone, sotalol, flecainide, and propafenone for the management of atrial fibrillation. Europace, 2011; 13: 329-345.

4. Piccini JP, Hasselblad V, Peterson ED et al. Comparative efficacy of dronedarone and amiodarone for the maintenance of sinus rhythm in patients with atrial fibrillation. J Am Coll Cardiol, 2009; 54: 1089-1095.

5. Zimetbaum P. Antiarrhythmic drug therapy for atrial fibrillation. Circulation, 2012; 125: 381-389. 
6. Fuster V, Rydén LE, Cannom DS et al. American College of Cardiology/American Heart Association Task Force on Practice Guidelines; European Society of Cardiology Committee for Practice Guidelines; European Heart Rhythm Association; Heart Rhythm Society. ACC/AHA/ESC 2006 Guidelines for the Management of Patients with Atrial Fibrillation: A report of the American College of Cardiology/American Heart Association Task Force on Practice Guidelines and the European Society of Cardiology Committee for Practice Guidelines (Writing Committee to Revise the 2001 Guidelines for the Management of Patients With Atrial Fibrillation): developed in collaboration with the European Heart Rhythm Association and the Heart Rhythm Society. Circulation, 2006; 114: e257-e354.

7. January CT, Wann LS, Alpert JS et al. 2014 AHA/ACC/HRS Guideline for the Management of Patients with Atrial Fibrillation: A Report of the American College of Cardiology/American Heart Association Task Force on Practice Guidelines and the Heart Rhythm Society. Circulation, 2014; 130: e199-e267.

8. Vaughan Williams EM. Classification of anti-arrhythmic drugs. In: Sandfte E, Flensted-Jensen E, Olesen KH eds. Symposium on cardiac arrhythmias. AB ASTRA, Södertälje, Sweden 1970; 449-472.

9. ICD-9-CM Guidelines, Conversion Table, and Addenda. Classification of Diseases, Functioning, and Disability. National Center for Health Statistics, CDC. (Accessed December 15, 2012, at http://www.cdc.gov/nchs/icd/icd9cm.htm).

10. Quan H, Sundararajan V, Halfon P et al. Coding algorithms for defining comorbidities in ICD-9-CM and ICD-10 administrative data. Med Care, 2005; 43: 1130-1139.
11. Lane DA, Lip GY. Use of the CHA(2)DS(2)-VASc and HAS-BLED scores to aid decision making for thromboprophylaxis in nonvalvular atrial fibrillation. Circulation, 2012; 126: 860-865.

12. Charlson ME, Pompei P, Ales KL et al. A new method of classifying prognostic comorbidity in longitudinal studies: Development and validation. J Chronic Dis, 1987; 40: 373-383.

13. Vorperian RV, Havighurst TC, Miller S et al. Adverse effects of low dose amiodarone: A meta-analysis. J Am Coll Cardiol, 1997; 30: 791-798.

14. Drug Approval Package: CORDARONE (amiodarone hydrochloride) Tablets. NDA \#018972. U.S. Food and Drug Administration. (Retrieved on November 10, 2014, at www.accessdata.fda. gov/drugsatfda_docs/nda/pre96/18-972_Cardarone.cfm).

15. Lafuente-Lafuente C, Longas-Tejero MA, Bergmann JF, Belmin J. Antiarrhythmics for maintaining sinus rhythm after cardioversion of atrial fibrillation. The Cochrane Library, 2012, Issue 5.

16. Roy D, Talajic M, Dorian P et al. Amiodarone to prevent recurrence of atrial fibrillation. Canadian Trial of Atrial Fibrillation Investigators. N Engl J Med, 2000; 342: 913-920.

17. Olshansky B, Sami M, Rubin A et al.; NHLBI AFFIRM Investigators. Use of amiodarone for atrial fibrillation in patients with preexisting pulmonary disease in the AFFIRM Study. Am J Cardiol, 2005; 95: 404-405.

18. AFFIRM First Antiarrhythmic Drug Substudy Investigators. Maintenance of sinus rhythm in patients with atrial fibrillation. An AFFIRM Substudy of the First Antiarrhythmic Drug. J Am Coll Cardiol, 2003; 42: 20-29.

19. Singh SN, Fisher SG, Deedwania PC et al. Pulmonary effect of amiodarone in patients with heart failure. J Am Coll Cardiol, 1997; 30: 514-517. 